\title{
Detection of biomolecules using optoelectronic biosensor based on localized surface plasmon resonance. Nanoimprint lithography approach
}

\author{
V. Chegel ${ }^{1}$, B. Lucas ${ }^{2}$, J. Guo ${ }^{2}$, A. Lopatynskyi ${ }^{1}$, O. Lopatynska ${ }^{3}$, L. Poperenko ${ }^{3}$ \\ ${ }^{1}$ V. Lashkaryov Institute of Semiconductor Physics, National Academy of Sciences of Ukraine, \\ 41, prospect Nauky, 03028 Kyiv, Ukraine, \\ Phone: +38 (044) 525-56-26, e-mail:vche111@yahoo.com, lop2000@ukr.net \\ ${ }^{2}$ The University of Michigan, 1301 Beal Avenue, Ann Arbor, MI 48109, USA, \\ E-mail:guo@umich.edu \\ ${ }^{3}$ Taras Shevchenko Kyiv National University, build. 1, 2, prospect Glushkova, 03022 Kyiv, Ukraine, \\ Phone: +38 (044) 526-22-96, e-mail: olga_lopatynska@ukr.net,plv@univ.kiev.ua
}

\begin{abstract}
In this work, we use a nanoimprint lithography fabrication technology to create uniformly-oriented and homogenous noble metal nanoparticle arrays with wellcontrolled size, shape and spacing, which can be the basis platform for the development of Localized Surface Plasmon Resonance (LSPR) sensors for biomolecular detection. Using this approach, we demonstrate proof-of-principle of an optical biosensor to quantify biomolecular interactions in a real-time mode using a UV-visible spectrophotometer. The sensor shows concentration-dependent kinetics of surface adsorption or binding of biomolecules and a capability to monitor antigen-antibody specific reactions, which is demonstrated by the reaction between bovine serum albumin (BSA) and anti-BSA immunoglobulin.
\end{abstract}

Keywords: localized surface plasmon resonance, nanoimprint lithography, biomolecular detection.

Manuscript received 10.12.08; accepted for publication 18.12.08; published online 10.03.08.

\section{Introduction}

Localized surface plasmon resonance (LSPR) phenomenon, as collective oscillation of free electrons in nanosized structures of high-conductive metals $[1,2]$, became widely attractive for the researchers since different methods of nanostructures fabrication had been developed, which give the opportunity to fabricate large nanoparticles arrays (NPAs) and nanostructured films $[3,4]$.

Numerous important applications of localized surface plasmon resonance phenomenon were demonstrated in recent years. Kreibig in 1995 [1] showed that the absorbance of an immobilized monolayer of gold colloids is sensitive to the refractive index of the surrounding solvent. Several experimental works related to influence of nanoparticle (NP) systems parameters (e.g. shape, size and spacing) on their extinction properties and observed optical dichroism were published recently [5-11].
Some basic methods of nanostructured film fabrication were demonstrated. Two of the most appropriate methods, NP deposition from the colloid solution and nanosphere lithography, were rather widely used for the investigation of fundamental properties of these systems [6, 12-14]. Van Duyne et al. [15], Natan et al. $[16,17]$ have shown that silver or gold nanoconstructs can be created using nanosphere lithography (NSL) on mica or glass surfaces to yield an optically detectable monolayer for the transduction of biomolecular binding events. G. Chumanov et al. [18] have shown the possibility of creating stable monolayers of silver nanoparticles, obtained from colloid solutions, using polymer layer that fixes low part of the nanoparticles and does not prevent the LSPR appearance on the surface of NP that is free of the polymer. Silver nanostructure arrays fabricated in such a way demonstrate extra narrow peaks in absorption spectra, which can be used to create high-sensitive biosensors. The major disadvantage of the mentioned technologies is 
their limitations associated with NP shape, monodispersity and reproducibility which adversely impact their viability for wide application.

In this work, we use a recently developed nanoimprint lithography technology to create uniformlyoriented and homogenous noble metal NPAs with wellcontrolled size, shape and spacing as a basis for the development of sensors for biomolecular detection. The most notable advantage of this method is the use of molds for the production of NP structures which makes nanoimprint lithography (NIL) a high-throughput and low-cost process capable for patterning large areas with sub-10 nm resolution capability $[19,20]$. This method provides a general platform for rapid prototyping of different types of NPAs (shapes, size, composition and spacing) and provides simultaneous fabrication over a billion monodisperse nanoparticles in a reproductive processing sequence directly on a variety of substrates. The use of a one-dimensional grating mold to produce two-dimensional patterns permits to obtain threedimensional nanostructures with a defined shape that form NPA with desired optical properties.

Among the wide-ranging applications of NPAs, biosensor application appears to be one of the most promising because of the possibility to detect interaction between biomolecules in the real-time mode [21, 22]. To produce sensor structure for the biomolecules detection, we have used NIL method for producing $\mathrm{Au}$ nanostructure arrays with parallelepiped shape, obtained from one-dimensional gratings. As a result, nanostructure arrays with well defined sizes $(120 \times 130 \times 50 \mathrm{~nm})$, shape and spacing $(230 \mathrm{~nm})$ were obtained. Using this approach, we demonstrate proof-ofprinciple of an optoelectronic biosensor to quantify biomolecular interactions in real time using a UV-visible spectrophotometer. Developed LSPR-sensor in its use has minimal technological requirements, namely, the availability of an UV-visible spectrophotometer and peristaltic pump. The sensor ability of biomolecular recognition relies on the change in the extinction spectrum of NPA as a function of biomolecular adsorption to the gold surface. Although nascent NILproduced nanoparticle arrays result in a nonselective sensor platform, a high degree of analyte selectivity can be conferred using the specificity of surface-attached ligands and passivation of the sensor surface to nonspecific binding [23, 24].

We believe this approach can be used to extend the fundamental research on LSPR and is applicable for commercialization of technology for producing LSPRbiochips.

\section{Theoretical background}

Noble metal nanoparticles possess the ability to support a localized surface plasmon resonance. The electric field distribution of the excited localized surface plasmon modes and their frequencies strongly depend on the size, shape and optical constants of the particle and the dielectric properties of the surrounding medium. In the simplest case of a spherical particle that is much smaller than the light wavelength, the polarizability of the particle is $[1,2]$

$\alpha=4 \pi \varepsilon_{0} R^{3} \frac{\varepsilon-\varepsilon_{m}}{\varepsilon+2 \varepsilon_{m}}$

where $\varepsilon_{0}$ is the dielectric permittivity of vacuum, $R$ is the nanoparticle radius, $\varepsilon$ is the dielectric function of the particle material and $\varepsilon_{m}$ is the dielectric function of the medium surrounding the particle. The excitation of the dipolar localized surface plasmon mode takes place, when the frequency of the incident light equals to the frequency that corresponds to the minimum of the denominator $\varepsilon+2 \varepsilon_{m}$ absolute value, which results in a maximum of the polarizability spectrum.

The occurrence of LSPR leads to an increase in the extinction of light by the nanoparticles. The simplest theoretical method for modeling the optical properties particularly, the extinction of nanoparticles - is the Mie theory for spherical particles. The extinction crosssection spectrum of the metallic sphere, the size of which is much smaller than the light wavelength, in the dipole approximation is described by the simplified Mie formula [1]:

$\sigma_{\text {ext }}(\omega)=9 \frac{\omega}{c} \varepsilon_{m}^{3 / 2} V_{p} \frac{\varepsilon_{2}(\omega)}{\left[\varepsilon_{1}(\omega)+2 \varepsilon_{m}\right]^{2}+\varepsilon_{2}(\omega)^{2}}$,

where $\omega$ is the light angular frequency, $c$ is the light velocity in vacuum, $V_{p}=\frac{4}{3} \pi R^{3}$ is the nanoparticle volume, $\varepsilon_{1}(\omega)$ and $\varepsilon_{2}(\omega)$ are the real and imaginary parts of the particle material dielectric function.

In the case of larger spherical nanoparticles, localized surface plasmon modes of higher multipole orders should be taken into account. In the framework of the Mie theory, the extinction cross-section of the spherical particle, accounting the multipole modes up to the order of $L$, is calculated using the following equations [1]:

$$
\begin{gathered}
\sigma_{e x t}=\frac{2 \pi}{|\vec{k}|^{2}} \sum_{l=1}^{L}(2 l+1) \operatorname{Re}\left(a_{l}+b_{l}\right), \\
a_{l}=\frac{m \psi_{l}(m x) \psi_{l}^{\prime}(x)-\psi_{l}^{\prime}(m x) \psi_{l}(x)}{m \psi_{l}(m x) \eta_{l}^{\prime}(x)-\psi_{l}^{\prime}(m x) \eta_{l}(x)}, \\
b_{l}=\frac{\psi_{l}(m x) \psi_{l}^{\prime}(x)-m \psi_{l}^{\prime}(m x) \psi_{l}(x)}{\psi_{l}(m x) \eta_{l}^{\prime}(x)-m \psi_{l}^{\prime}(m x) \eta_{l}(x)},
\end{gathered}
$$

where $\vec{k}$ is the wavevector of light in surrounding medium, $m=n / n_{m}, n$ is the complex refractive index of particle material, $n_{m}$ is the real refractive index of surrounding medium, $x=|\vec{k}| R$ is the size parameter, 
$\psi_{l}(z)$ and $\eta_{l}(z)$ are Riccati-Bessel functions. The prime indicates differentiation with respect to the argument of the function.

To estimate the LSPR properties of nanoparticles in the shape of parallelepiped, one should consider the contribution of all localized surface plasmon modes that are excited by the incident light. The resulting polarizability is calculated as the sum over these modes labeled by $i[1]$ :

$$
\alpha=\varepsilon_{0} V \sum_{i=1}^{N_{i}} \frac{C_{i}\left(\varepsilon-\varepsilon_{m}\right)}{\varepsilon_{m}+L_{i}\left(\varepsilon-\varepsilon_{m}\right)},
$$

where $V$ is the volume of the particle, $C_{i}$ are the oscillator strengths and $L_{i}$ are the geometrical depolarization factors.

To simplify this approach, the use of analytical methods for calculation of nanoparticle extinction properties is preferable. One of the promising methods is the Green function formalism in its analytical approach [25] or in the frame of the quasi-crystalline approximation (QCA) of the theory of multiple scattering of waves [26], which allows accounting the interparticle interaction. However, the both mentioned methods have some disadvantages, because the first one does not take into account the interaction between nanoparticles, whereas the second one can characterize only spherical (ellipsoidal) objects. The known discrete dipole approximation (DDA) method [27] does not have these disadvantages; however, due to requirement of supercomputer resources we do not consider it. The detailed comparison and possible application of these techniques will be considered in the next work.

\section{Mold and sample fabrication}

\subsection{Mold fabrication.}

A thin $10 \mathrm{~nm}$ film of $\mathrm{Cr}$ serving as the reactive ion etching (RIE) mask layer was electron beam evaporated on $200 \mathrm{~nm}$ thermally grown $\mathrm{SiO}_{2}$. The nanoimprint resist

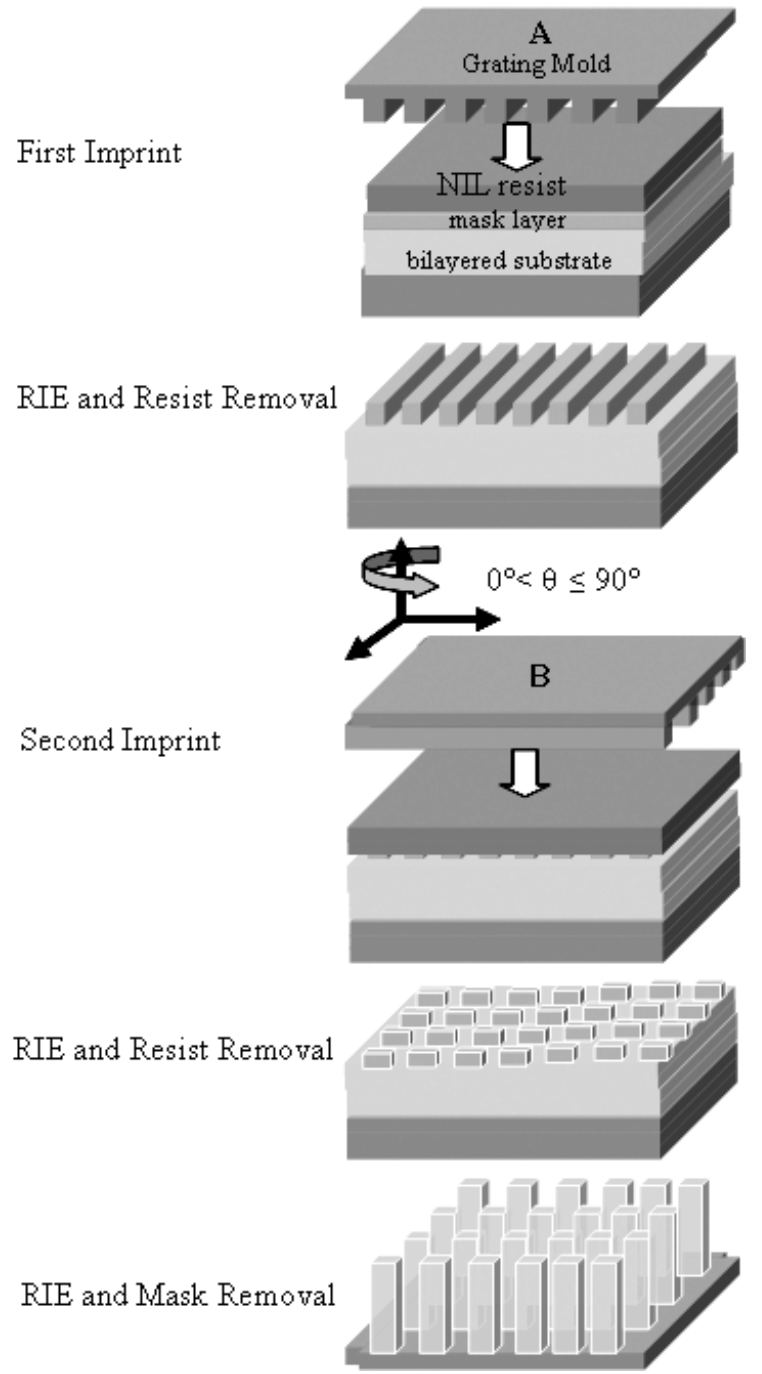

Fig. 1. The general approach to creation of molds that are subsequently used to fabricate NPAs.

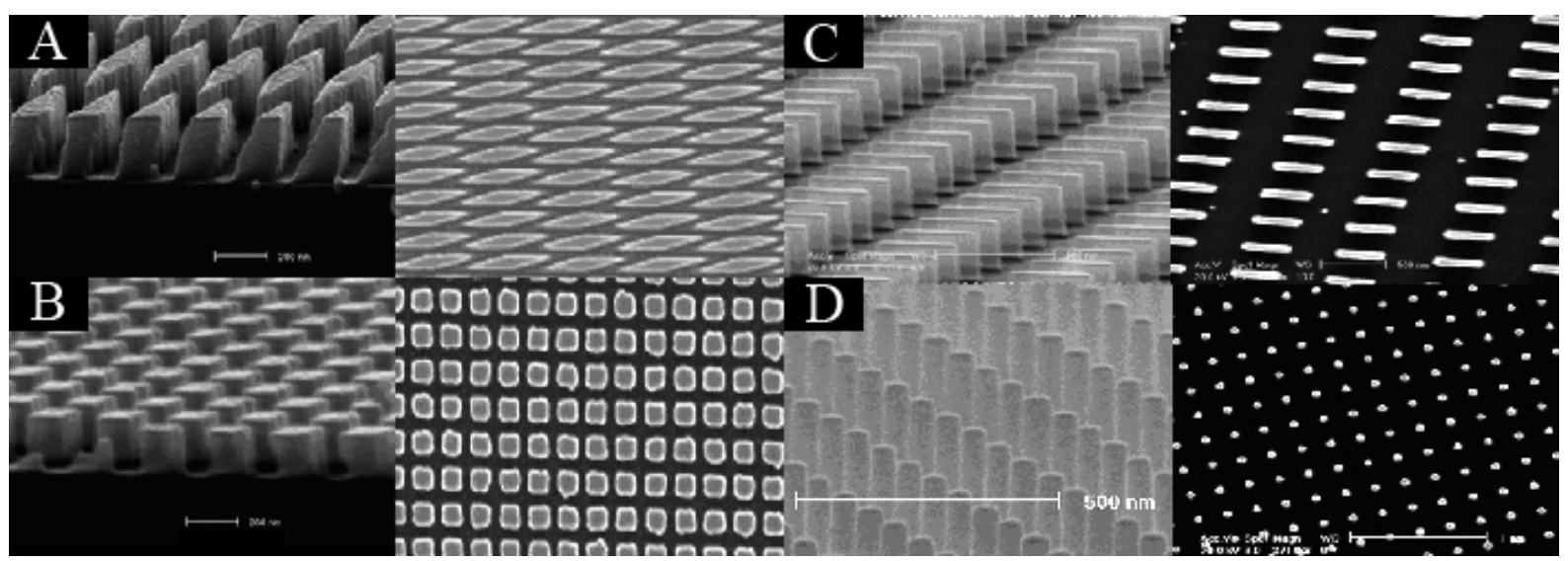

Fig. 2. Different NPAs created using NIL fabrication. 
(MicroResist MR-I 8020) was spincoated onto the substrate to the appropriate thickness (determined by mold depth, duty cycle and period), baked on hotplate $\left(140{ }^{\circ} \mathrm{C} ; 5 \mathrm{~min}\right)$ to remove residual solvent and imprinted in a custom-built nanoimprinter $(4.6 \mathrm{MPa}$; $\left.5 \mathrm{~min} ; 180^{\circ} \mathrm{C}\right)$. After separation of the mold and substrate, the residual polymer was removed in $\mathrm{O}_{2}$ plasma and the pattern was transferred to the $\mathrm{Cr}$ layer using RIE $\left(\mathrm{Cl}_{2} / \mathrm{O}_{2} ; 200 \mathrm{~W}\right)$. The resist was stripped using acetone incubation, rinsed with methanol and isopropanol and dried using a stream of $\mathrm{N}_{2}$ gas. The above process steps were repeated for the second imprint and the $200 \mathrm{~nm} \mathrm{SiO}_{2}$ was subsequently reactive-ion etched $\left(\mathrm{CHF}_{3} ; 150 \mathrm{~W}\right)$ using the patterned $\mathrm{Cr}$ mask. After etching, the $\mathrm{Cr}$ mask was removed using a wet chromium etchant (CR-14; Cyantek Co.), rinsed with a copious amount of deionized water $\left(\mathrm{DI}: \mathrm{H}_{2} \mathrm{O}\right)$ and dried with $\mathrm{N}_{2}$. The mold was then prepared for imprinting by vapor-phase surfactant treatment to reduce moldpolymer adhesion.

The process illustrated in Fig. 1 describes the general approach for creation of large-area molds that are used to fabricate NPAs afterwards. Nanostructured molds are utilized through usual NIL processing steps that include imprinting, residual polymer removal, metallization and lift-off. This technique can be used to create a variety of structures by simply using different one-dimensional gratings (i.e. with various duty cycles or periods) and relative angular orientation of the gratings for successive imprints. The examples of the application of described method are shown in Fig. 2, where the same grating mold is used to create a lattice of square and diamond-shaped pillars (Fig. 2a, b), while structures presented in Fig. $2 \mathrm{c}, \mathrm{d}$ are produced by application of different grating molds.

\subsection{Sample fabrication.}

Pyrex glass substrates with the thickness $0.5 \mathrm{~mm}$ $(n=1.474)$ were cleaned in a $1: 1$ piranha solution $\left(30 \% \mathrm{H}_{2} \mathrm{O}_{2}: 29 \% \mathrm{NH}_{4} \mathrm{OH}\right)$, rinsed with a copious amount of DI: $\mathrm{H}_{2} \mathrm{O}$ and dried using $\mathrm{N}_{2}$. The resist was spincoated to the appropriate thickness on the substrate, baked on a hotplate for solvent removal and imprinted. After nanoimprinting and sample separation, the residual polymer layer of the imprinted MR-I resist was removed using $\mathrm{O}_{2}$ plasma reactive-ion etching. Metallization was accomplished using an electron beam evaporator by first depositing a $3 \mathrm{~nm}$ Ti adhesion layer followed by $50 \mathrm{~nm}$ of $\mathrm{Au}$. Lift-off was performed by soaking the sample in acetone and using a low-power ultrasonic bath. After completion of lift-off, samples were rinsed with methanol and IPA and dried with $\mathrm{N}_{2}$. The AFM images and section of $120 \times 130 \times 50 \mathrm{~nm}$ Au nanorectangles with interparticle distance equal to $237 \mathrm{~nm}$ are depicted in Fig. 3. It should be noted that AFM technique doesn't resolve the correct estimation of the distance between NPAs due to limitation related to the geometry of AFM tip.

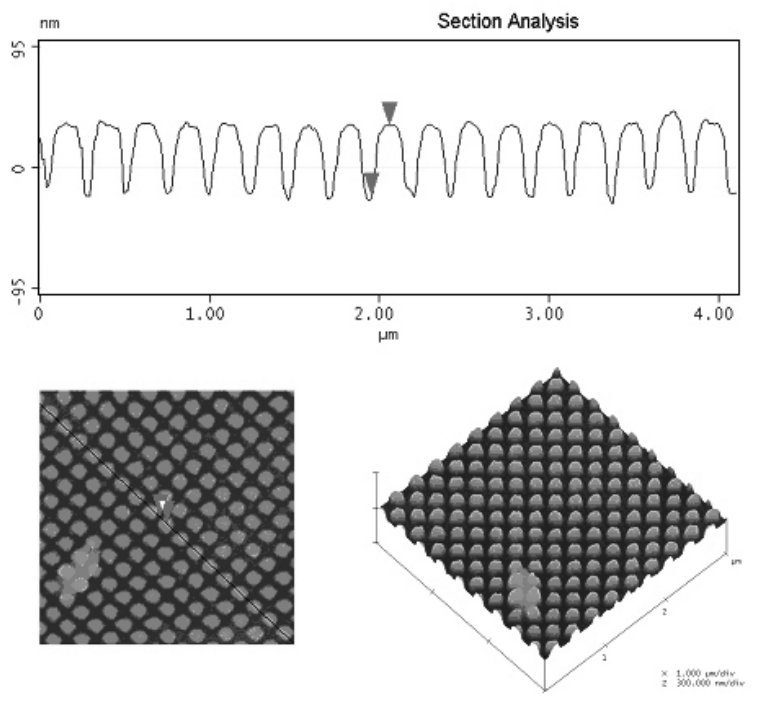

Fig. 3. AFM images and section of used Au NPAs.

\section{Experimental methods}

All extinction measurements were taken using a Nikon TE300 Eclipse inverted microscope $(20 \times$ objective with the numerical aperture 0.44) with transmitted light coupled into an Ocean Optics SD2000 fiber-coupled spectrometer using an achromatic lens (experimental setup is presented schematically in Fig. 4). For polarization microextinction measurements, a linear polarizer mounted in a rotating module was aligned to the optic axis of the microscope directly above the sample stage. Proper alignment was verified by matching extinction spectra at polarizer positions that were $180^{\circ}$ apart. All measurements were taken using normally incident light and referenced to unpatterned glass. A refractive index change was introduced by embedding the NPA in a DI: $\mathrm{H}_{2} \mathrm{O}$ water. Experiments were performed using the original LSPR sensor chip, wherein pyrex glass substrate (size about of $2 \times 2 \mathrm{~mm}$ ) modified with layer of NPAs was fixed on the surface of microscopic glass substrate (size $25 \times 76 \mathrm{~mm}$ ) and covered by a transparent $20 \mu \mathrm{l}$ flow cell. For the real-time measurements of biomolecular interactions the peristaltic pump (Multiflex, USA, flow rate $10 \mu \mathrm{l} / \mathrm{min}$ ) was used. The glycerol, phosphate buffer saline (PBS) solution $(\mathrm{pH}=7.4)$, bovine serum albumin (BSA), anti-BSA immunoglobulin (IgG) were purchased from Sigma-Aldrich.

\section{Results and discussion}

The nanoscale biosensors based on LSPR spectroscopy operate in the same manner as their SPR counterparts by transducing small changes in refractive index near the noble metal surface into LSPR response (a measurable wavelength shift response). 


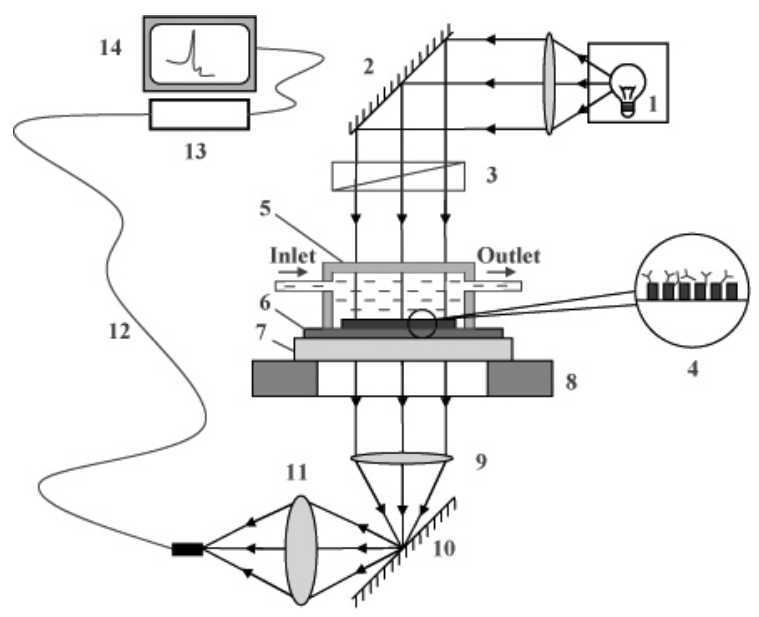

Fig. 4. Schematic presentation of the experimental set-up for extinction spectroscopy measurements. $1-100 \mathrm{~W}$ halogen source; 2,10- mirrors; 3 - polarizer; $4-$ surface nanostructures with a biomolecular layer: rectangular array with adsorbed IgG molecules; 5 - flow cell; 6 - glass-ceramic substrate; 7 - glass substrate; 8 - sample holder; 9, 11 - lenses; 12 - fiber-optic cable; 13 - spectrometer; 14 - computer.

The biosensors are liquid sensors that work in the realtime mode; therefore, first we tested NPA structures for their sensitivity to changes of the liquid environment refractive index. The glycerol-water solutions with various refractive indices were used for this aim. The refractive indices of these solutions were determined using the standard Abbe refractometer.Measurements for the detection of refractive index changes were carried out in a flowing regime, with real-time monitoring of the wavelength (initial $=650 \mathrm{~nm}$ ) shift. The results obtained for calibrated glycerol solutions clearly show that the optical extinction of the NPA structures is sensitive to the refractive index of the surrounding liquid medium. The results of the experiment are depicted in Fig. 5. Close to linear dependence of the extinction shift on the refractive index was observed (see inset for Fig. 5). The measurements were performed for the right side of the extinction peak $(\lambda=660 \mathrm{~nm})$. It should be noted that we found a correlation between the chosen wavelength on our peak and the resulting response; however, during experiments there were some peculiarities related to the difference in a rate and direction of the wavelength shift for different points in the extinction peak branches. From our observations, it is most preferable to use wavelengths found on the right side of the peak, however, this point requires additional study.

The issue of the sensitivity of LSPR biosensors is rather complicated due to a confluence of many factors which include the electrodynamic interaction between nanostructures that decreases with an increase of interparticle distance and influences the shape as well as spectral position of the LSPR peak [26]. The nanorectangle NPA used in this study is characterized by in-plane lateral dimensions of $120 \mathrm{~nm}$ and $130 \mathrm{~nm}$, uniformly oriented on a square lattice with a period of $237 \mathrm{~nm}$. Even with such a minuscule anisotropy in these near-unity aspect ratio NPA constituents, the dichroism is readily observed (Fig. 6) and the polarization dependent sensitivity was followed. This can imply a significant difference in the character of the electric field in the vicinity of the nanoparticles for different polarizations of the light source. This variation in LSPR response demonstrates that polarized light can be used for the optimization of the parameters of LSPR sensors produced by NIL.

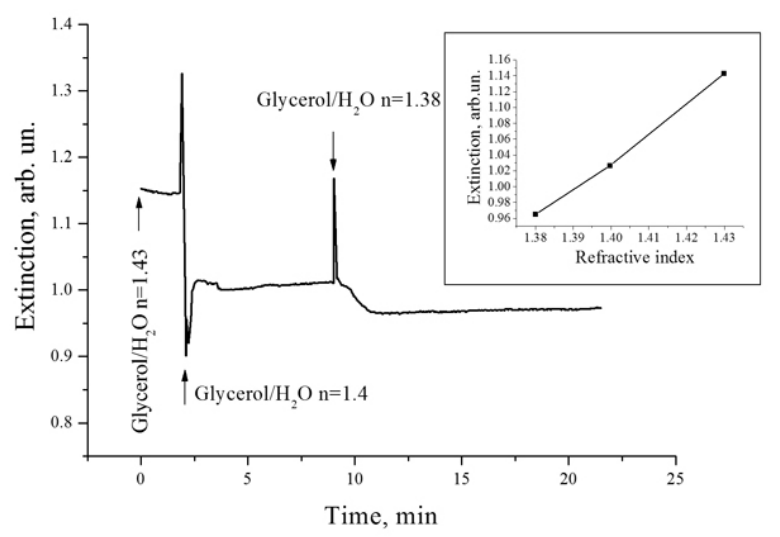

Fig. 5. The real-time kinetic dependence of extinction at the change of refractive index in the liquid environment. Inset: dependence of extinction on the refractive index.

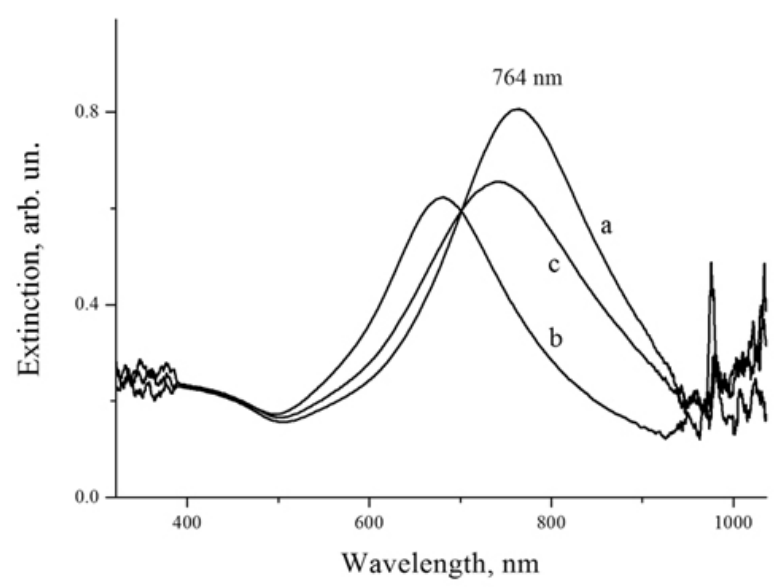

Fig. 6. Polarization extinction spectra for the nanoblock NPA with linear polarization of the incident light oriented along (a) the long axis $\left(0^{\circ}\right.$, longitudinal mode) and (b) short axis of the nanoblocks $\left(90^{\circ}\right.$, transverse mode). (c) The extinction spectrum for the case of $45^{\circ}$ polarization.

Similar to conventional SPR, the wavelength position and shape of LSPR extinction peak influence the sensitivity of biosensor utilizing the plasmon resonance phenomenon. It is known that a more narrow peak, positioned at shorter wavelength, exhibits the lower sensitivity $S=\Delta \lambda / \Delta n$, where $\lambda$ is the peak center wavelength and $n$ is the refractive index of the 
environment. However, due to necessity of computer treatments of extreme region in extinction spectra to define the exact wavelength peak position, a narrow peak appears as more suitable to obtain the lower "noise" of kinetic curve. Oppositely, the wide wavelength peak exhibits higher sensitivity, but, at the same time, a higher noise level. From this point of view, 3D-geometry and density of NPA on the surface of substrate should be optimized. The factor of polarization for NPAs is also important due to easy possibility to produce the asymmetric structures with high dichroism degree.

To estimate the kinetic response of LSPR biosensor on adsorption of real biological materials, we utilized the produced NPAs to detect the biospecific reaction between bovine serum albumin (BSA, MW $\approx 60 \mathrm{kDa}$ ) and anti-BSA immunoglobulin ( $\mathrm{IgG}, \mathrm{MW} \approx 150 \mathrm{kDa})$. The results of experiment are depicted in Fig. 7, where the realtime kinetic dependence of extinction for the specific reaction between BSA and immunoglobulin dissolved in PBS buffer solution with different concentrations of the PBS/anti-BSA solution is shown. As one can see from Figs 7 and 8, at the beginning of the experiment the comparatively strong extinction shift was obtained for the PBS/BSA solution of $500 \mu \mathrm{g} / \mathrm{ml}$ concentration and, further, concentration dependent wavelength shift for IgG solutions beginning from $0.1 \mu \mathrm{g} / \mathrm{ml}$. It is evident that a distinct response due to BSA-anti-BSA specific reaction arises at the injection of anti-BSA solution with concentration of $0.5 \mu \mathrm{g} / \mathrm{ml}$. It should be noted that the smallest $(0.1 \mu \mathrm{g} / \mathrm{ml})$ concentration was not detected; this proves our assumption that the LSPR biosensor can be more suitable for registration of biomolecules with small molecular mass due to more developed Au surface comparatively with that in conventional SPR sensors and, respectively, due to possibility for small biomolecules, which easily penetrate and are adsorbed onto lateral faces, to fill the volume between nanostructures.

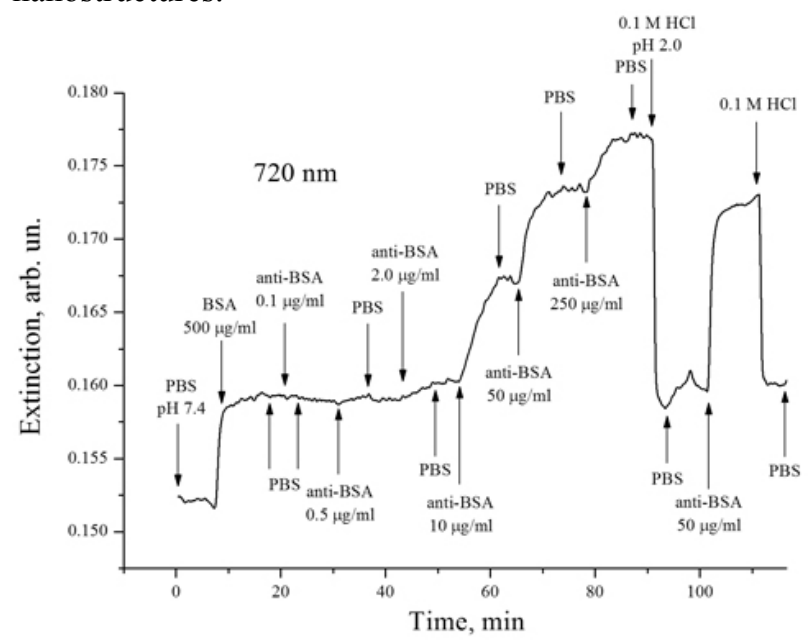

Fig. 7. The real-time kinetic dependence of extinction for the specific reaction BSA-anti-BSA with different concentrations of the PBS/anti-BSA immunoglobulin solution.
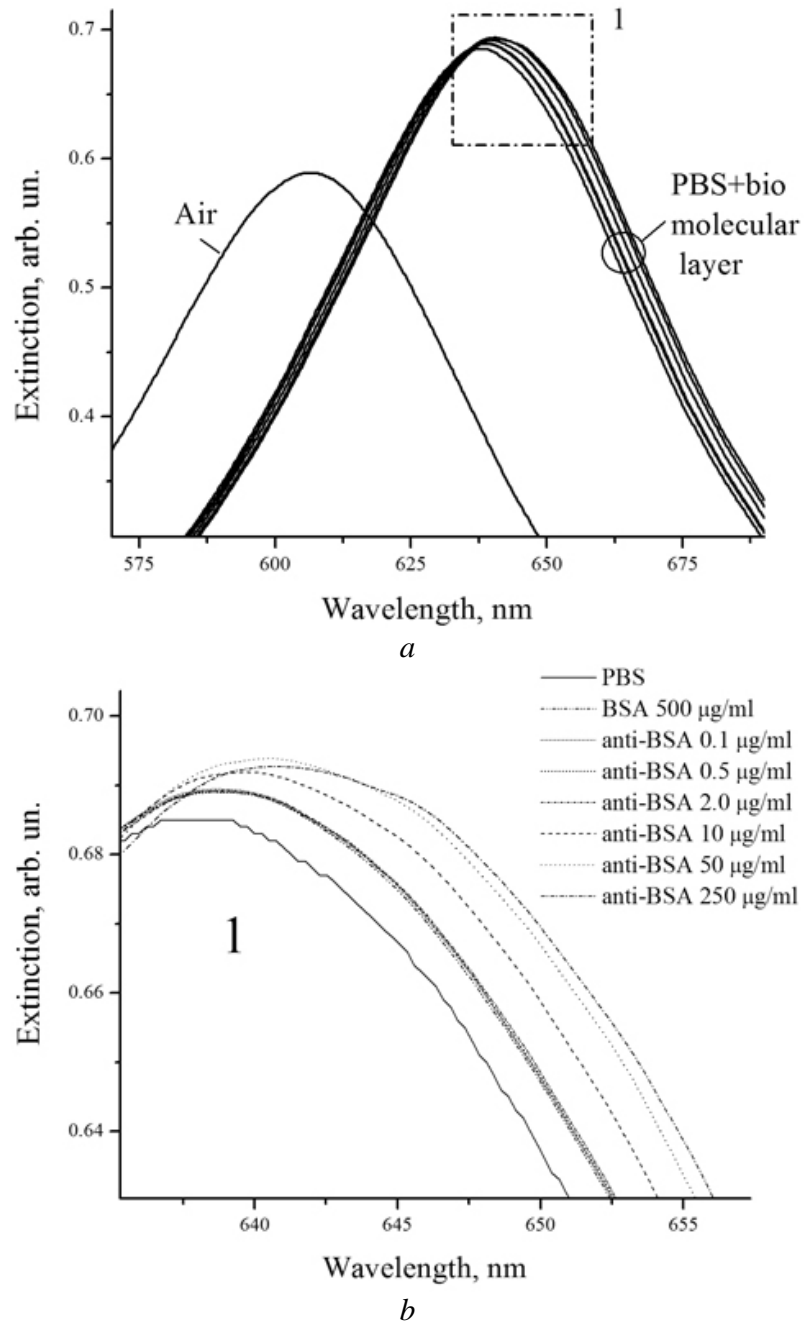

Fig. 8. (a) Extinction spectra for NPAs in air, in buffer solution and for various concentrations of PBS/anti-BSA immunoglobulin solution. (b) Magnified part of the spectral range 1.

In addition, a high degree of specificity of the reaction was observed during the experiment after washing the $\operatorname{IgG}$ layer with $0.1 \mathrm{M} \mathrm{HCl}$ solution, when close to $100 \%$ of antibody was removed, whereas BSA layer remained completely at the sensor surface. As evident from the abovementioned sensogram, the developed LSPR biosensor is completely able for regeneration and repeating the specific reaction.

\section{Conclusion}

A novel developed LSPR biosensor based on approach to fabricate large-area, uniformly-oriented noble metal NPAs using nanoimprint lithography was presented. With the increasing number of applications based on the field enhancements provided by metal NPs, we believe this method can be practically important for the serial production of homogenous nanostructure populations and biosensors based on them. The experimental and theoretical studies of NIL-produced noble metal NPAs 
possessing a variety of unique dimensional characteristics and related LSPR attributes are ongoing. $\mathrm{Au}$ nanostructure NPA capability for detection of adsorption of biomolecules with different molecular weight in a real-time mode has been demonstrated in this work. The performed experiments show promising perspectives of the LSPR method for exploitation of NPA-structures as the basis for biomolecular detection and monitoring of specific biomolecular interactions.

\section{Acknowlegements}

This work was supported by NATO CLG grant PDD(CP0-(CBP.NUKR.CLG981776). The authors are grateful to Dr. O. Lytvyn for AFM measurements.

\section{References}

1. 1. U. Kreibig, M. Vollmer, Optical Properties of Metal Clusters. Springer-Verlag, Berlin, Heidelberg, 1995.

2. E.F. Venger, A.V. Goncharenko, M.L. Dmitruk, Optics of Small Particles and Disperse Media. Naukova dumka, Kyiv, 1999 (in Ukrainian).

3. S.A. Maier, Plasmonics - Towards subwavelength optical devices // Current Nanoscience 1(1), p. 1722 (2005).

4. H.A. Atwater et al., The new "p-n junction": Plasmonics enables photonic access to the nanoworld // MRS Bulletin 30(5), p. 385-389 (2005).

5. J.J. Mock, D.R. Smith and S. Schultz, Local refractive index dependence of plasmon resonance spectra from individual nanoparticles // Nano Letters 3(4), p. 485-491 (2003).

6. J.J. Mock et al., Shape effects in plasmon resonance of individual colloidal silver nanoparticles // J. Chem. Phys. 116(15), p. 6755-6759 (2002).

7. K.H. Su et al., Interparticle coupling effects on plasmon resonances of nanogold particles // Nano Letters 3(8), p. 1087-1090 (2003).

8. M.D. Malinsky et al., Nanosphere lithography: Effect of substrate on the localized surface plasmon resonance spectrum of silver nanoparticles // $J$. Phys. Chem. B 105(12), p. 2343-2350 (2001).

9. Y. Dirix et al., Oriented pearl-necklace arrays of metallic nanoparticles in polymers: A new route toward polarization-dependent color filters // Advanced Materials 11(3), p. 223-227 (1999).

10. C.L. Haynes and R.P. Van Duyne, Dichroic optical properties of extended nanostructures fabricated using angle-resolved nanosphere lithography // Nano Letters 3(7), p. 939-943 (2003).

11. B.K. Canfield et al., Remarkable polarization sensitivity of gold nanoparticle arrays // Appl. Phys. Lett. 86(18), p. 183109-183111 (2005).

12. S.J. Oldenburg et al., Light scattering from dipole and quadrupole nanoshell antennas // Appl. Phys. Lett. 75(8), p. 1063-1065 (1999).
13. L.J. Sherry et al., Localized surface plasmon resonance spectroscopy of single silver nanocubes // Nano Letters 5(10), p. 2034-2038 (2005).

14. A. Bouhelier et al., Electromagnetic interactions in plasmonic nanoparticle arrays // J. Phys. Chem. B 109(8), p. 3195-3198 (2005).

15. J.C. Riboh, A.J. Haes, A.D. McFarland, C. Ranjit Yonzon, R.P. Van Duyne, A nanoscale optical biosensor: Real-time immunoassay in physiological buffer enabled by improved nanoparticle adhesion // J. Phys. Chem. B 107(8), p. 1772-1780 (2003).

16. K.C. Grabar, R.G. Freeman, M.B. Hommer, M.J. Natan, Preparation and characterization of $\mathrm{Au}$ colloid monolayers // Anal. Chem. 67(4), p. 735-743 (1995).

17. R.G. Freeman, K.C. Grabar, K.J. Allison et al., Selfassembled metal colloid monolayers: An approach to SERS substrates // Science 267(5204), p. 16291632 (1995).

18. G. Chumanov, S. Malinich, Coupled planar silver nanoparticle arrays as refractive index sensors // J. Opt. A: Pure Appl. Opt. 8, p. 144-147 (2006).

19. S.Y. Chou and P.R. Krauss, Imprint lithography with sub-10 nm feature size and high throughput // Microelectronic Eng. 35(1-4), p. 237-240 (1997).

20. M.T. Li et al., Pattern transfer fidelity of nanoimprint lithography on six-inch wafers // Nanotechnology 14(1), p. 33-36 (2003).

21. A.D. McFarland and R.P. Van Duyne, Single silver nanoparticles as real-time optical sensors with zeptomole sensitivity // Nano Letters 3(8), p. 1057 1062 (2003).

22. N. Nath and A. Chilkoti, A colorimetric gold nanoparticle sensor to interrogate biomolecular interactions in real time on a surface // Analyt. Chem. 74(3), p. 504-509 (2002).

23. P. Schuck, Use of surface plasmon resonance to probe the equilibrium and dynamic aspects of interactions between biological macromolecules // Annu. Rev. Biophys. Biomol. Struct. 26, p. 541-566 (1997).

24. W. Knoll, Interfaces and thin films as seen by bound electromagnetic waves // Annu. Rev. Phys. Chem. 49, p. 569-638 (1998).

25. V. Lozovski, Electrodynamical interactions inside a system of nano-particles // Physica E 19, p. 263-277 (2003).

26. S.M. Kachan and A.N. Ponyavina, Spectral properties of close-packed monolayers consisting of metal nanospheres // J. Phys.: Condens. Matter 14(1), p. 103-111 (2002).

27. W.H. Yang, G.C. Schatz, R.P. Van Duyne, Discrete dipole approximation for calculating extinction and Raman intensities for small particles with arbitrary shapes // J. Chem. Phys. 103, p. 869-875 (1995). 